\title{
ON FINITE MODIFICATIONS OF ALGEBROID SURFACES
}

\author{
Dedicated to Professor Yukio Kusunoki on his 60th birthday
}

\author{
By KiYOshi NirNo
}

\section{$\S 1$. Introduction.}

Let $R$ be an open Riemann surface, $\mathfrak{M}(R)$ the family of non-constant meromorphic functions on $R$ and $P(f)$ the number of values which are not taken by $f \in \mathfrak{M}(R)$. We denote by $P(R)$ the Picard constant of $R$ defined by

$$
P(R)=\sup \{P(f) ; f \in \mathfrak{M}(R)\} .
$$

In general we have $P(R) \geqq 2$. The significant meaning of this Picard constant lies in the following fact:

Theorem A (Ozawa [9]). If $P(R)<P(S)$ for another Riemann surface $S$, then there is no non-trivial analytic mapping of $R$ into $S$.

From now on we shall confine ourselves to finitely sheeted covering algebroid surfaces defined as proper existence domains of algebroid functions. From the theory of algebroid functions we have $P\left(R_{n}\right) \leqq 2 n$ for an $n$-sheeted algebroid surface $R_{n}$. An $n$-sheeted algebroid surface $R_{n}$ is called regularly branched when it has no branched point other than those of order $n-1$.

Let $\xi_{n}$ be the family of entire functions having an infinite number of zeros whose orders are coprime to $n$ and $\mathbb{E}_{n}^{*}$ the subfamily of $\mathbb{E}_{n}$ consisting of entire functions orders of all zeros of which are less than $n$.

We denote by $R_{n}$ and $\widetilde{R}_{n}$ two algebroid surfaces defined by $y^{n}=G(z)$ and $y^{n}=\widetilde{G}(z)$, respectively, where $G(z)$ and $\tilde{G}(z)$ belong to $\mathbb{E}_{n}^{*}$. If $G(z)$ has the same zeros with the same multiplicity as $\tilde{G}(z)$ in $|z| \geqq r_{0}$ for a suitable positive number $r_{0}$ and has at least one distinct zero with the multiplicity from $\tilde{G}(z)$ in $|z|<r_{0}$, then we call $\tilde{R}_{n}$ a finite modification of $R_{n}$ (cf. Ozawa [11]).

We now consider two $n$-sheeted, regularly branched algebroid surfaces $R_{n}$ and $\widetilde{R}_{n}$ and two $m$-sheeted, regularly branched algebroid surfaces $S_{m}$ and $\widetilde{S}_{m}$. Suppose that $P\left(R_{n}\right)=2 n, P\left(S_{m}\right)=2 m$ and $\widetilde{R}_{n}$ and $\widetilde{S}_{m}$ are finite modifications of $R_{n}$ and $S_{m}$, respectively. In our previous paper [8] we had a perfect condition for the existence of analytic mappings of $R_{n}$ into $S_{m}$ and investigated the structure of the family $\mathscr{S}_{2}\left(R_{n}, S_{m}\right)$ of projections of analytic mappings of $R_{n}$ into $S_{m}$.

Received September 11, 1984 
In the present paper we shall consider the following two problems:

(A) What is $P\left(\widetilde{R}_{n}\right)$ ?

(B) Are there any analytic mappings among $R_{n}, \widetilde{R}_{n}, S_{m}$ and $\widetilde{S}_{m}$ ?

And we shall obtain generalizations of results of the author [6].

We assume that the reader is familiar with the Nevanlinna theory of meromorphic functions and the usual notations such as $T(r, f), N(r, a, f), \bar{N}(r, a, f)$, $m(r, f)$ etc. (see e.g. [5]).

\section{§2. A functional equation.}

For our purpose we have to consider a functional equation. We firstly prove

THEOREM 1. Let $m$ be a positive integer. Suppose that two non-constant entire functions $H(z)$ and $M(z)$ with $H(0)=M(0)=0$ and four integers $a, b, c$ and $d$ with $0<a \leqq b<m$ and $0<c \leqq d<m$ satisfy the following functional equation

$$
\left(e^{M(z)}-\gamma\right)^{a}\left(e^{M(z)}-\delta\right)^{b}=F(z)\left(e^{H(z)}-\sigma\right)^{c}\left(e^{H(z)}-\tau\right)^{d}
$$

with four constants $\gamma, \delta, \sigma$ and $\tau$ and a meromorphic function $F(z)=f_{1}(z)^{m} f_{2}(z)$, where $f_{1}(z)$ and $f_{2}(z)$ are meromorphic in $|z|<+\infty$ and

$$
T\left(r, f_{2}\right)=o\left(T\left(r, e^{M}\right)\right) \quad \text { or } \quad T\left(r, f_{2}\right)=o\left(T\left(r, e^{H}\right)\right) \quad r \rightarrow \infty
$$

outside a set of $r$ of finite measure. Then we hane

and

( I ) $a=c$ and $b=d$

(II) one of the following four cases:

$$
\begin{aligned}
& H(z)=M(z), \quad F(z)=1, \quad \gamma=\sigma, \quad \delta=\tau, \\
& H(z)=M(z), \quad F(z)=1, \quad \gamma=\tau, \quad \delta=\sigma, \\
& H(z)=-M(z), \quad F(z)=(-1)^{a+b} \gamma^{a} \delta^{b} e^{(a+b) M(z)}, \quad \gamma \sigma=\delta \tau=1, \\
& H(z)=-M(z), \quad F(z)=(-1)^{a+b} \gamma^{a} \delta^{b} e^{(a+b) M(z)}, \quad \gamma \tau=\delta \sigma=1 .
\end{aligned}
$$

(2.4) and (2.6) may occur in the case $a=b$ only.

To prove our Theorem 1 we need

LEMMA A ([10]). Let $H(z)$ be a non-constant entire function and $\alpha$ a non-zero constant. Then we have

$$
N_{2}\left(r, 0, e^{H}-\alpha\right) \sim m(r, f) \quad \text { and } \quad N_{1}\left(r, 0, e^{H}-\alpha\right)=o\left(m\left(r, e^{H}\right)\right) \quad r \rightarrow \infty
$$

outside a set of $r$ of finite measure, where $N_{2}(r, 0, f)$ is the counting function of 
simple zeros of the function $f$ and $N_{1}(r, 0, f)=N(r, 0, f)-\bar{N}(r, 0, f)$.

Proof of Theorem 1. Firstly we prove that the condition

$$
T\left(r, f_{2}\right)=o\left(T\left(r, e^{M}\right)\right) \quad r \rightarrow \infty
$$

outside a set of $r$ of finite measure is equivalent to the condition

$$
T\left(r, f_{2}\right)=o\left(T\left(r, e^{H}\right)\right) \quad r \rightarrow \infty
$$

outside a set of $r$ of finite measure. Assume that the condition (2.7) is true. Then we consider simple zeros of $\left(e^{M}-\gamma\right)\left(e^{M}-\delta\right)$. It follows from the functional equation (2.1) that these are zoros of $f_{2}$ or $\left(e^{H}-\sigma\right)^{c}\left(e^{H}-\tau\right)^{d}$, and consequently

$$
N_{2}\left(r, 0,\left(e^{M}-\gamma\right)\left(e^{M}-\delta\right)\right) \leqq N\left(r, 0, f_{2}\right)+N\left(r, 0,\left(e^{H}-\sigma\right)^{c}\left(e^{H}-\tau\right)^{d}\right) .
$$

Hence Lemma A and (2.7) imply

$$
(2+o(1)) m\left(r, e^{M}\right) \leqq o\left(m\left(r, e^{M}\right)\right)+(c+d+o(1)) m\left(r, e^{H}\right) \quad r \rightarrow \infty
$$

and so

$$
m\left(r, e^{M}\right)=O\left(m\left(r, e^{H}\right)\right) \quad r \rightarrow \infty
$$

outside a set of $r$ of finite measure. Thus we obtain (2.8). Conversely we assume that the condition (2.8) holds. Then from (2.1) we have

$$
N_{2}\left(r, 0,\left(e^{H}-\sigma\right)\left(e^{H}-\tau\right)\right) \leqq N\left(r, 0, f_{2}\right)+N\left(r, 0,\left(e^{M}-\gamma\right)^{a}\left(e^{M}-\delta\right)^{b}\right) .
$$

Hence it follows from Lemma A and (2.8) that

$$
(2+o(1)) m\left(r, e^{H}\right) \leqq o\left(m\left(r, e^{H}\right)\right)+(a+b+o(1)) m\left(r, e^{M}\right) \quad r \rightarrow \infty
$$

and so

$$
m\left(r, e^{H}\right)=O\left(m\left(r, e^{M}\right)\right) \quad r \rightarrow \infty
$$

outside a set of $r$ of finite measure. Hence we have (2.7).

Thus we see that the condition (2.2) can be replace by

$$
T\left(r, f_{2}\right)=o\left(m\left(r, e^{M}\right)\right) \quad \text { and } T\left(r, f_{2}\right)=o\left(m\left(r, e^{H}\right)\right) \quad r \rightarrow \infty
$$

outside a set of $r$ of finite measure. Further we can deduce from the above discussion that

$$
m\left(r, e^{H}\right)=O\left(m\left(r, e^{M}\right)\right) \text { and } m\left(r, e^{M}\right)=O\left(m\left(r, e^{H}\right)\right) \quad r \rightarrow \infty
$$

outside a set of $r$ of finite measure.

We now prove ( I ). Assume that $a<c$. The functional equation (2.1) implies that a simple zero $z_{1}$ of $e^{M}-\gamma$ is a zero of order $a$ of $G(z) \equiv F(z)\left(e^{H(z)}-\sigma\right)^{c}$ $\left(e^{H(z)}-\tau\right)^{d}$. It follows from our assumption of $F(z)$ and $a<c \leqq b<m$ that $z_{1}$ is a zero of $f_{2}$ or a pole of $f_{2}$ or a multiple zero of $\left(e^{H}-\sigma\right)\left(e^{H}-\tau\right)$. Hence Lemma $\mathrm{A}$ and (2.9) yield 


$$
m\left(r, e^{M}\right)=o\left(m\left(r, e^{H}\right)\right) \quad r \rightarrow \infty
$$

outside a set of $r$ of finite measure, which contradicts (2.10). We next assume that $a>c$. Then we deduce from (2.1) that a simple zero of $e^{H}-\sigma$ is a zero of $1 / f_{2}$ or a pole of $1 / f_{2}$ or a multiple zero of $\left(e^{M}-\gamma\right)\left(e^{M}-\delta\right)$. Hence Lemma A and (2.9) imply

$$
m\left(r, e^{H}\right)=o\left(m\left(r, e^{M}\right)\right) \quad r \rightarrow \infty
$$

outside a set of $r$ of finite measure, which contradicts (2.10). Therefore we obtain $a=c$. Similarly considering simple zeros of $e^{M}-\delta$ and $e^{H}-\tau$ and taking Lemma A, (2.9) and (2.10) into account we have $b=d$. Thus (I) is proved.

From (2.1) and (I) we have

$$
\begin{aligned}
& \left(e^{M(z)}-\gamma\right)^{a}\left(e^{M(z)}-\delta\right)^{b}=F(z)\left(e^{H(z)}-\sigma\right)^{a}\left(e^{H(z)}-\tau\right)^{b}, \\
& H(z) \not \equiv \text { const., } \quad M(z) \neq \text { const., } \quad H(0)=M(0)=0, \quad F(z)=f_{1}(z)^{m} f_{2}(z), \\
& 0<a \leqq b<m, \quad \gamma \delta \sigma \tau(\gamma-\delta)(\sigma-\tau) \neq 0 .
\end{aligned}
$$

Considering simple zeros of $e^{M}-\gamma$ and $e^{H}-\sigma$, we can deduce from Lemma A and (2.9) that

$$
m\left(r, e^{M}\right) \sim m\left(r, e^{H}\right) \quad r \rightarrow \infty,
$$

that is, $m\left(r, e^{M}\right) / m\left(r, e^{H}\right) \rightarrow 1$ as $r \rightarrow \infty$, outside a set of $r$ of finite measure. Further we have

and

$$
T(r, F)=O\left(T\left(r, e^{M}\right)+T\left(r, e^{H}\right)\right)=O\left(m\left(r, e^{H}\right)\right) \quad r \rightarrow \infty
$$

$$
\begin{aligned}
N\left(r, \infty, F^{\prime} / F\right) \leqq & N(r, 0, F)+N(r, \infty, F) \\
\leqq & N_{1}\left(r, 0, e^{M}-r\right)+N_{1}\left(r, 0, e^{M}-\delta\right)+N_{1}\left(r, 0, e^{H}-\sigma\right) \\
& +N_{1}\left(r, 0, e^{H}-\tau\right)+N\left(r, 0, f_{2}\right)+N\left(r, \infty, f_{2}\right)+O(\log r) \\
= & o\left(m\left(r, e^{M}\right)+m\left(r, e^{H}\right)\right)=o\left(m\left(r, e^{H}\right)\right)
\end{aligned}
$$

outside a set of $r$ of finite measure. Hence we obtain

$$
\begin{aligned}
T\left(r, F^{\prime} / F\right) & =m\left(r, F^{\prime} / F\right)+N\left(r, \infty, F^{\prime} / F\right) \\
& \leqq O(\log r T(r, F))+N\left(r, \infty, F^{\prime} / F\right) \\
& =o\left(m\left(r, e^{H}\right)\right),
\end{aligned}
$$

and consequently

$$
T\left(r, F^{\prime} / F\right)=o\left(m\left(r, e^{H}\right)\right) \quad r \rightarrow \infty
$$

outside a set of $r$ of finite measure. Since (2.12) and (2.13) valid, the proof of Theorem in [7] can be transferred to our case, even if $a+b \neq m$. Thus the 
proof of (II) follows the lines of that of Theorem in [7, pp. 298-301].

\section{§3. Known results.}

Further we need some known results.

THEOREM A ([1]). Let $R_{n}$ be an $n$-sheeted regularly branched algebroid surface with $P\left(R_{n}\right)>(3 / 2) n$. Then we have $P\left(R_{n}\right)=2 n$.

THEOREM B ([1], [8]). Let $R_{n}$ be a regularly branched algebroid surface defined by $y^{n}=G(z)\left(G \in\left(E_{n}^{*}\right)\right.$. If $P\left(R_{n}\right)=2 n$, then $G(z)$ satisfies the following functional equation.

$$
\begin{aligned}
& G(z)=f(z)^{n}\left(e^{H(z)}-\alpha\right)^{k}\left(e^{H(z)}-\beta\right)^{n-k}, \\
& H(z) \neq \text { const. }, \quad H(0)=0, \quad \alpha \beta(\alpha-\beta) \neq 0, \quad(k, n)=1, \quad 1 \leqq k \leqq n / 2,
\end{aligned}
$$

where $H(z)$ is entire, $f(z)$ is meromorphic, $\alpha$ and $\beta$ two complex constants and $k$ is an integer.

THEOREM C ([3], [4], [8]). Let $R_{n}$ and $S_{m}$ be two algebroid surfaces defined by $y^{n}=G(z)$ and $u^{m}=g(w)\left(G, g \in\left(\xi_{n}\right)\right.$, respectively and further $G(z)$ satisfies the inequality with a constant $\eta$

$$
\frac{N_{n}^{*}(r, 0, G)}{N(r, 0, G)} \geqq \eta>0
$$

for a set of $r$ of infinite measure, where $N_{n}^{*}(r, 0, G)$ is the counting function of zeros whose orders are coprime to $n$. If there is an analytic mapping $\phi$ of $R_{n}$ into $S_{m}$, then $n=p m$ with a positive integer $p$ and the projection $h(z)$ of $\phi$ is a single-valued entire function of $z$ and satisfies

$$
g(h(z))=f(z)^{m} G(z)^{k}, \quad p \leqq k p \leqq n-1,
$$

where $f(z)$ is a suitable meromorphic function and $k$ is a suitable positive integer which is coprime to $m$.

Conversely, if $n=p m$ with a positive integer $p$ and there is an entire function $h(z)$ satisfies (3.2) with a suitable meromorphic function $f(z)$ and a suitable positive integer $k$ which is coprime to $m$, there exists an analytic mapping of $R_{n}$ into $S_{m}$ whose projection is $h(z)$.

\section{$\S 4$. Picard constants.}

With respect to the problem (A) we have the following

THEOREM 2. Let $R_{n}$ and $\widetilde{R}_{n}$ be two $n$-sheeted regularly branched algebroid surfaces defined by $y^{n}=G(z)$ and $y^{n}=\tilde{G}(z)$, respectively, where $G(z)$ and $\tilde{G}(z)$ are 
two entire functions belonging to $\xi_{n}^{*}$. If $P\left(R_{n}\right)=2 n$ and $\widetilde{R}_{n}$ is a finite modification of $R_{n}$, then we have $P\left(\widetilde{R}_{n}\right) \leqq(3 / 2) n$.

Proof. By the definition of finite modifications of algebroid surfaces we have

$$
\tilde{G}(z)=Q(z) G(z),
$$

where $Q(z)$ is a rational function satisfying the following conditions (M1)-(M6) (Hereafter in this case we simply say that $Q(z)$ satisfies the condition (M) with respect to $G(z)$ and $n)$ :

(M1) $Q(z)$ has a form

$$
Q(z)=\prod_{\imath=1}^{\mu}\left(z-a_{\imath}\right)^{\mu_{i}} \prod_{\jmath=1}^{\nu}\left(z-b_{\jmath}\right)^{-\nu_{j}} .
$$

(M2) $\mu, \mu_{\imath}, \nu, \nu$, are non-negative integers and $\mu+\nu \geqq 1$.

(M3) $a_{\imath}$ and $b_{3}$ are mutually distinct constants and their moduli are less than $r_{0}$.

(M4) If $a_{i}$ is not a zero of $G(z)$, then $0<\mu_{i}<n$ and $\left(\mu_{i}, n\right)=1$.

(M5) If $a_{\imath}$ is a zero of order $k_{\imath}$ of $G(z)$, then $k_{i}+\mu_{i}<n$ and $\left(k_{i}+\mu_{\imath}, n\right)=1$.

(M6) $b_{j}$ is a zero of order $l_{j}$ of $G(z)$ satisfying $l_{j}-\nu_{j}=0$ or $0<l_{j}-\nu_{j}<n, \quad\left(l_{j}-\right.$ $\left.\nu_{\jmath}, n\right)=1$.

Since $P\left(R_{n}\right)=2 n$, Theorem B implies that $G(z)$ satisfies

$$
\begin{aligned}
& G(z)=f_{1}(z)^{n}\left(e^{H(z)}-\alpha\right)^{l}\left(e^{H(z)}-\beta\right)^{n-l}, \\
& H(z) \equiv \text { const., } \quad H(0)=0, \quad \alpha \beta(\alpha-\beta) \neq 0, \quad(l, n)=1, \quad 1 \leqq l \leqq n / 2,
\end{aligned}
$$

where $H(z)$ is an entire function, $f_{1}(z)$ is a meromorphic function, $\alpha$ and $\beta$ are two complex constants and $l$ is an integer.

Now suppose, to the contrary, that $P\left(R_{n}\right)>(3 / 2) n$. Then it follows from Theorem A and Theorem B that $\tilde{G}(z)$ satisfies

$$
\begin{aligned}
& \tilde{G}(z)=f_{2}(z)^{n}\left(e^{M(z)}-\gamma\right)^{k}\left(e^{M(z)}-\delta\right)^{n-k}, \\
& M(z) \neq \text { const. }, \quad M(0)=0, \quad \gamma \delta(\gamma-\delta) \neq 0, \quad(k, n)=1, \quad 1 \leqq k \leqq n / 2,
\end{aligned}
$$

where $M(z)$ is entire, $f_{2}(z)$ is meromorphic and $\gamma$ and $\delta$ are two constants. It follows from (4.1), (4.2) and (4.3) that

$$
\left(e^{M(z)}-\gamma\right)^{k}\left(e^{M(z)}-\delta\right)^{n-k}=Q(z)\left\{f_{1}(z) f_{2}(z)^{-1}\right\}^{n}\left(e^{H(z)}-\alpha\right)^{l}\left(e^{H(z)}-\beta\right)^{n-l} .
$$

Since $Q(z)$ is a rational function, we have $T(r, Q)=o\left(T\left(r, e^{M}\right)\right)$ as $r \rightarrow \infty$. Hence our Theorem 1 yields that

$$
Q(z)\left\{f_{1}(z) f_{2}(z)^{-1}\right\}^{n}=1 \text { or }=(-1)^{n} \gamma^{k} \delta^{k} e^{n M(z)},
$$

which contradicts the condition $(\mathrm{M})$. Therefore we have $P\left(\widetilde{R}_{n}\right) \leqq(3 / 2) n$. 


\section{$\S 5$. Existence of analytic mappings.}

Let $R_{n}$ and $S_{m}$ be two regularly branched algebroid surfaces defined by $y^{n}=G(z)$ and $u^{m}=g(w)$ with $G(z) \in \xi_{n}^{*}$ and $g(w) \in F_{m}^{*}$, respectively. If $P\left(R_{n}\right)=2 n$ and $P\left(S_{m}\right)=2 m$, then it follows from Theorem B that $G(z)$ and $g(w)$ satisfy

and

$$
\begin{aligned}
& G(z)=F(z)^{n}\left(e^{H(z)}-\alpha\right)^{l}\left(e^{H(z)}-\beta\right)^{n-l}, \\
& H(0)=0, \quad \alpha \beta(\alpha-\beta) \neq 0, \quad(l, n)=1, \quad 1 \leqq l \leqq n / 2
\end{aligned}
$$

$$
\begin{aligned}
& g(w)=f(w)^{m}\left(e^{L(w)}-\gamma\right)^{k}\left(e^{L(w)}-\delta\right)^{m-k}, \\
& L(0)=0, \quad \gamma \delta(\gamma-\delta) \neq 0, \quad(k, m)=1, \quad 1 \leqq k \leqq m / 2,
\end{aligned}
$$

where $H$ and $L$ are two non-constant entire functions, $F$ and $f$ are two meromorphic functions, $l$ and $k$ are two integers and $\alpha, \beta, \gamma$ and $\delta$ are four complex constants. Further let $\widetilde{R}_{n}$ and $\widetilde{S}_{m}$ be finite modifications of $R_{n}$ and $S_{m}$ defined by $y^{n}=\tilde{G}(z)$ and $u^{m}=\tilde{g}(w)$ with $\tilde{G}(z)=Q(z) G(z) \in \mathfrak{F}_{n}^{*}$ and $g(w)=\tilde{q}(w) g(w) \in\left(\mathfrak{F}_{m}^{*}\right.$, respectively, where

$$
Q(z)=\prod_{\imath=1}^{\mu}\left(z-a_{\imath}\right)^{\mu} \prod_{j=1}^{\nu}\left(z-b_{j}\right)^{-\nu}
$$

and

$$
q(w)=\prod_{\imath=1}^{\sigma}\left(w-a_{\imath}\right)^{\sigma_{i}} \prod_{j=1}^{\tau}\left(w-d_{j}\right)^{-\tau}
$$

satisfy the condition (M) with respect to $G(z)$ and $n$ and with respect to $g(w)$ and $m$, respectively.

Now in this section we consider the problem (B), that is, whether there exist analytic mappings among $R_{n}, \widetilde{R}_{n}, S_{m}$ and $\widetilde{S}_{m}$. We have already obtained a perfect condition for the existence of analytic mappings of $R_{n}$ into $S_{m}$ in [8]. Using Lemma A we here note that $G, \tilde{G}, g$ and $\tilde{g}$ satisfy the condition (3.1) in Theorem $\mathrm{C}$ and consequently we can apply Theorem $\mathrm{C}$ to analytic mappings in this section.

Firstly we have

THEOREM 3. There exists an analytic mapping $\phi$ of $R_{n}$ into $\widetilde{S}_{m}$ if and only if $n=p m$ with a positive integer $p$ and there exist an entire function $h(z)$ and meromorphic functions $f_{1}^{*}(z)$ and $f_{2}^{*}(z)$ satisfying one of the following equations:
(a) $\quad H(z)=L(h(z))-L(h(0)), \quad q(h(z))=f_{1}^{*}(z)^{m}, \quad \gamma / \alpha=\delta / \beta=e^{L(h(0))}$,
(a') $H(z)=L(h(z))-L(h(0)), \quad q(h(z))=f_{1}^{*}(z)^{m}, \quad \gamma / \beta=\delta / \alpha=e^{L(h(0))}$,
(b) $\quad H(z)=-L(h(z))+L(h(0)), \quad q(h(z))=f_{2}^{*}(z)^{m}, \quad \gamma \alpha=\delta \beta=e^{L(h(0))}$,
( $\left.\mathrm{b}^{\prime}\right) \quad H(z)=-L(h(z))+L(h(0)), \quad q(h(z))=f_{2}^{*}(z)^{m}, \quad \gamma \beta=\delta \alpha=e^{L(h(0))}$. 
Proof. Suppose that there is an analytic mapping $\phi$ of $R_{n}$ into $\widetilde{S}_{m}$. Then it follows from Theorem $C$ that $n=p m$ with a positive integer $p$ and the projection $h(z)$ of $\phi$ is a single-valued entire function of $z$ and satisfies

$$
\tilde{g}(h(z))=f_{3}(z)^{m} G(z)^{\lambda},
$$

where $f_{3}(z)$ is a meromorphic function and an integer $\lambda$ satisfies $(\lambda, m)=1$ and $p \leqq p \lambda \leqq n-1$. We put $l \lambda=a m+c$ and $(n-l) \lambda=b m+d$, where $a, b, c$ and $d$ are four integers satisfying $0 \leqq c \leqq m-1,0 \leqq d \leqq m-1$. Then we have $c>0$ and $d>0$ because of $(\lambda, m)=1$ and $(l, n)=1$. From (5.1), (5.2), (5.4) and (5.5) we have

$$
\left(e^{M(z)}-\gamma e^{-L(h(0))}\right)^{k}\left(e^{M(z)}-\delta e^{-L(h(0))}\right)^{m-k}=F_{1}(z)\left(e^{H(z)}-\alpha\right)^{c}\left(e^{H(z)}-\beta\right)^{d},
$$

where $M(z)=L(h(z))-L(h(0)), F_{1}(z)=f_{1}(z)^{m} f_{2}(z), f_{2}(z)=q(h(z))^{-1}$ and $f_{1}(z)=e^{-L(h(0))}$ $f(h(z))^{-1} f_{3}(z) F(z)^{p \lambda}\left(e^{H(z)}-\alpha\right)^{a}\left(e^{H(z)}-\beta\right)^{b}$. Since $q(z)$ is rational, we have $T(r, q(h))$ $=O(T(r, h))$ as $r \rightarrow \infty$. Since $e^{L(z)}$ is transcendental, Theorem 2 in Clunie [2] implies $T(r, h)=o\left(T\left(r, e^{L(h)}\right)\right)$ and consequently

$$
T\left(r, f_{2}\right)=T(r, q(h))=o\left(T\left(r, e^{L(h)}\right)\right)=o\left(T\left(r, e^{M}\right)\right) \quad r \rightarrow \infty .
$$

Hence $f_{2}(z)$ satisfies the condition (2.2) in our Theorem 1. Applying Theorem 1 to functional equation (5.6) we have one of the following four cases:

$$
\begin{aligned}
& H(z)=M(z), \quad F_{1}(z)=1, \quad \gamma e^{-L(h(0))}=\alpha, \quad \delta e^{-L(h(0))}=\beta, \\
& H(z)=M(z), \quad F_{1}(z)=1, \quad \gamma e^{-L(h(0))}=\beta, \quad \gamma e^{-L(h(0))}=\alpha, \\
& H(z)=-M(z), \quad F_{1}(z)=(-1)^{m} \gamma^{k} \delta^{m-k} e^{m(M(z)-L(h(0)))}, \quad \gamma \alpha=\delta \beta=e^{L(h(0))}, \\
& H(z)=-M(z), \quad F_{1}(z)=(-1)^{m} \gamma^{m-k} \delta^{k} e^{m(M(z)-L(h(0)))}, \quad \gamma \beta=\delta \alpha=e^{L(h(0))},
\end{aligned}
$$

which correspond, respectively, to (a), $\left(a^{\prime}\right),(b)$ and $\left(b^{\prime}\right)$ in our Theorem 3 with $f_{1}^{*}(z)=f_{1}(z)$ in (a) and $\left(\mathrm{a}^{\prime}\right)$ and $f_{2}^{*}(z)=-\gamma^{k / m} \delta^{(m-k) / m} f_{1}(z) e^{L(h(z))-2 L(h(0))}$ in (b) and $\left(b^{\prime}\right)$.

Conversely, suppose that $n=p m$ with a positive integer $p$ and there is an entire function $h(z)$ satisfying one of the four cases (a), (a'), (b) and $\left(\mathrm{b}^{\prime}\right)$. Firstly we note that for positive integers $n, m, p, l$ and $k$ satisfying $n=p m,(l, n)=$ $(k, m)=1$ there are integers $\lambda, a, b, \rho, c$ and $d$ satisfying

and

$$
l \lambda+a m=k, \quad(n-l) \lambda+b m=m-k, \quad(\lambda, m)=1, \quad 1 \leqq \lambda \leqq m-1
$$

$$
l \rho+c m=m-k, \quad(n-l) \rho+d m=k, \quad(\rho, m)=1, \quad 1 \leqq \rho \leqq m-1 .
$$

If $h(z)$ satisfies (a), then we have

$$
\begin{aligned}
\tilde{g}(h(z)) & =q(h(z)) f(h(z))^{m}\left(e^{L(h(z))}-\gamma\right)^{k}\left(e^{L(h(z))}-\delta\right)^{m-k} \\
& =f_{1}^{*}(z)^{m} f(h(z))^{m} e^{m L(h(0))}\left(e^{H(z)}-\alpha\right)^{l \lambda+a m}\left(e^{H(z)}-\beta\right)^{(n-l) \lambda+b m} \\
& =f_{1}(z)^{m}\left\{F(z)^{n}\left(e^{H(z)}-\alpha\right)^{l}\left(e^{H(z)}-\beta\right)^{n-l}\right\}^{\lambda}
\end{aligned}
$$




$$
=f_{1}(z)^{m} G(z)^{\lambda} \text {, }
$$

where $f_{1}(z)=e^{L(h(0))} f_{1}^{*}(z) f(h(z)) F(z)^{-p \lambda}\left(e^{H(z)}-\alpha\right)^{a}\left(e^{H(z)}-\beta\right)^{b}$. Similarly we have

$$
\begin{aligned}
& \tilde{g}(h(z))=f_{2}(z)^{m} G(z)^{\rho}, \\
& f_{2}(z)=e^{L(h(0))} f_{1}^{*}(z) f(h(z)) F(z)^{-\rho \rho}\left(e^{H(z)}-\alpha\right)^{c}\left(e^{H(z)}-\beta\right)^{d}
\end{aligned}
$$

if $\left(a^{\prime}\right)$ is the case, or

$$
\begin{aligned}
& \tilde{g}(h(z))=f_{3}(z)^{m} G(z)^{\lambda} \\
& f_{3}(z)=-\gamma^{k / m} \delta^{(m-k) / m} f_{2}^{*}(z) f(h(z)) e^{-H(z)} F(z)^{-p \grave{i}}\left(e^{H(z)}-\alpha\right)^{a}\left(e^{H(z)}-\beta\right)^{b}
\end{aligned}
$$

if (b) is the case, or

$$
\begin{aligned}
& \tilde{g}(h(z))=f_{4}(z)^{m} G(z)^{\rho} \\
& f_{4}(z)=-\gamma^{k / m} \delta^{(m-k) / m} f_{2}^{*}(z) f(h(z)) e^{-H(z)} F(z)^{-p \rho}\left(e^{H(z)}-\alpha\right)^{c}\left(e^{H(z)}-\beta\right)^{d}
\end{aligned}
$$

if $\left(b^{\prime}\right)$ is the case. Hence from Theorem $C$ there is an analytic mapping $\phi$ of $R_{n}$ into $\widetilde{S}_{m}$ whose projection is $h(z)$.

Thus the proof of our Theorem 3 is complete.

As an immediate consequence of our Theorem 3 and Theorem 2 in [8] we have

COROLLARY 1. If there is an analytic mapping $\psi$ of $R_{n}$ into $\widetilde{S}_{m}$, then there exists an analytic mapping of $R_{n}$ into $S_{m}$ whose projection is the same $h(z)$ as that of $\psi$.

If we take $\widetilde{R}_{n}$ as $\widetilde{S}_{m}$ in Theorem 3 , then we have $H(z)= \pm H(h(z)) \mp H(h(0))$ and consequently $h(z)$ is a linear function $A z+B(A \neq 0)$. Hence there is no meromorphic function $f^{*}(z)$ satisfying $f^{*}(z)^{n}=Q(A z+B)$ because of (5.3) and the condition (M). Therefore from Theorem 3 we obtain

COROLLARY 2. There is no non-trivial analytic mapping of $R_{n}$ into $\widetilde{R}_{n}$.

From the arguments in the proof of Theorem 3 we can deduce

THEOREM 4. There exists an analytic mapping of $\tilde{R}_{n}$ into $\tilde{S}_{m}$ if and only if there exist an entire function $h(z)$, two meromorphic functions $f_{1}^{*}(z)$ and $f_{2}^{*}(z)$ and two positive integers $p$ and $\lambda$ such that $n=p m,(\lambda, m)=1, p \leqq p \lambda \leqq n-1$ and one of the following equations holds:

(a) $\quad H(z)=L(h(z))-L(h(0)), \quad q(h(z))=f_{1}^{*}(z)^{m} Q(z)^{\lambda}$,

$$
\frac{\gamma}{\alpha}=\frac{\delta}{\beta}=e^{L(h(0))} \quad \text { or } \quad \frac{\gamma}{\beta}=\frac{\delta}{\alpha}=e^{L(h(0))} \text {, }
$$

(b) $\quad H(z)=-L(h(z))+L(h(0)), \quad q(h(z))=f_{2}^{*}(z)^{m} Q(z)^{\lambda}$, 


$$
\gamma \alpha=\delta \beta=e^{L(h(0))} \text { or } \gamma \beta=\delta \alpha=e^{L(h(0))} \text {. }
$$

It follows from our Theorem 4 and Theorem 2 in [8] that

COROLlARY 3. If there is an analytic mapping $\phi$ of $\tilde{R}_{n}$ into $\tilde{S}_{m}$, then there exists an analytic mapping of $R_{n}$ into $S_{m}$ whose projection $i s$ the same $h(z)$ as that of $\psi$.

We can also deduce

THEOREM 5. There exists an analytıc mapping of $\tilde{R}_{n}$ into $S_{m}$ if and only if there exist an entire function $h(z)$, two meromorphic functions $f_{1}^{*}(z)$ and $f_{2}^{*}(z)$ and two positive integers $p$ and $\lambda$ such that $n=p m,(\lambda, m)=1, p \leqq p \lambda \leqq n-1$ and one of the following equations holds

(a) $\quad H(z)=L(h(z))-L(h(0)), \quad Q(z)^{\lambda}=f_{1}^{*}(z)^{m}$,

$$
\frac{\gamma}{\alpha}=\frac{\delta}{\beta} e^{L(h(0))} \quad \text { or } \quad \frac{\gamma}{\beta}=\frac{\delta}{\alpha} e^{L(h(0))} \text {, }
$$

$$
\begin{aligned}
& H(z)=-L(h(z))+L(h(0)), \quad Q(z)^{\lambda}=f_{2}^{*}(z)^{m}, \\
& \gamma \alpha=\delta \beta=e^{L(h(0))} \quad \text { or } \quad \gamma \beta=\delta \alpha=e^{L(h(0))} .
\end{aligned}
$$

COROLLARY 4. If there is an analytic mapping $\phi$ of $\tilde{R}_{n}$ into $S_{m}$, then there exists an analytic mapping of $R_{n}$ into $S_{m}$ whose projection is the same $h(z)$ as that of $\psi$.

Now we shall give an example which shows existence of an analytic mapping of $\widetilde{R}_{n}$ into $S_{m}$.

EXAMPLE. $\quad n=8, m=4$. Put $G(z)=\left(e^{2 z}-1\right)\left(e^{2 z}+1\right)^{7}, Q(z)=z^{4} /(z-\pi i / 2)^{4}, \quad \tilde{G}(z)$ $=Q(z) G(z)$ and $g(w)=\left(e^{w}-1\right)\left(e^{w}+1\right)^{3}$. Let $R_{8}, \tilde{R}_{8}$ and $S_{4}$ be algebroid surfaces defined by $y^{8}=G(z), y^{8}=\tilde{G}(z)$ and $u^{4}=g(w)$, respectively. Then since $z=0$ is a zero of order 5 of $\tilde{G}(z)$ and $z=\pi i / 2$ is a zero of order 3 of $\tilde{G}(z)$, it is clear that these surfaces are regularly branched with $P\left(R_{8}\right)=16$ and $P\left(S_{4}\right)=8$ (cf. Theorem B), $\widetilde{R}_{8}$ is a finite modification of $R_{8}$ and satisfy (a) of Theorem 5 with

$$
\begin{aligned}
& H(z)=2 z, \quad L(w)=w, \quad h(z)=2 z, \quad f_{1}^{*}(z)=z /(z-\pi i / 2), \\
& \lambda=1, \quad \gamma=\alpha=1, \quad \delta=\beta=-1 .
\end{aligned}
$$

Thus we see that there exists an analytic mapping of $\tilde{R}_{8}$ into $S_{4}$. However we suppose that $\left(\mu_{\imath}, n\right)=\left(\nu_{\jmath}, n\right)=1$ in (5.3). Then since $(\lambda, m)=1$ and $n=p m$, we have $\left(\lambda \mu_{\imath}, m\right)=\left(\lambda_{\nu}, m\right)=1$ and so there is no meromorphic function $f^{*}(z)$ satisfying $Q(z)^{\lambda}=f^{*}(z)^{m}$. Hence we finally deduce from Theorem 5 that

COROLlary 5. Suppose that $\left(\mu_{\imath}, n\right)=\left(\nu_{\jmath}, n\right)=1$ in (5.3). Then there is no 
analytic mapping of $\widetilde{R}_{n}$ into $S_{m}$.

\title{
REFERENCES
}

[1] Aogal, H., Picard constant of a finitely sheeted covering surfaces. Kōdai Math. Sem. Rep. 25 (1973), 219-224.

[2] Clunie, J., The composition of entire and meromorphic functions. Mathematical Essays dedicated to A. J. Macintyre (Ohio Univ. Press, 1970), 75-92.

[3] Hiromi, G., ANd H. Mutō, On existence of analytic mappings, II. Kōdai Math. Sem. Rep. 19 (1967), 439-450.

[4] Mutō, H., On analytic mappings among algebroid surfaces. Kōdai Math. Sem. Rep. 21 (1969), 191-204.

[5] Nevanlinna, R., Analytic functions. Springer-Verlag, (1970), pp. 373.

[6] Nino, K., On finite modifications of two- or three-sheeted covering open Riemann surfaces. Kōdai Math. Sem. Rep. 19 (1967), 415-424.

[7] NiINo, K., On the functional equation $\left(e^{M}-\gamma\right)^{k}\left(e^{M}-\delta\right)^{m-k}=f^{m}\left(e^{H}-\sigma\right)\left(e^{H}-\tau\right)^{m-k}$. Aequationes Math. 22 (1981), 293-301.

[8] Nino, K., On analytic mappings between two algebroid surfaces. Complex Variables Theory Appl. 2 (1983-84), 283-293.

[9] Ozawa, M., On complex analytic mappings. Kōdai Math. Sem. Rep. 17 (1965), 93-102.

[10] Ozawa, M., On ultrahyperelliptic surfaces. Kōdai Math. Sem. Rep. 17 (1965), 103-108.

[11] OzAWA, M., On a finite modification of an ultrahyperelliptic surfaces. Kōdai Math. Sem. Rep. 19 (1967), 312-316.

\author{
FACULTY OF TECHNOLOGY \\ KANAZAWA UNIVERSITY \\ 2-40-20, Kodatsuno \\ KANAZAWA 920 \\ JAPAN
}

